\title{
THE STRUCTURE OF SPECTRAL OPERATORS WITH COMPLETELY CONTINUOUS IMAGINARY PART ${ }^{1}$
}

\author{
NOBORU SUZUKI
}

J. T. Schwartz [4] has established a structure theorem for a spectral operator on Hilbert space whose imaginary part is completely continuous. The purpose of this note is to give a new proof of this theorem which is based on the algebraic approach to the theory of nonselfadjoint operators developed by the author [5], and throughout this proof we shall show how the algebraic approach may be used to technical advantage in the study of nonselfadjoint operators on Hilbert space.

Actually, Schwartz proved the structure theorem for a spectral operator $A$ satisfying the condition that $A A^{*}-A^{*} A$ is completely continuous, but we shall concentrate our attention on the case when $A$ has the completely continuous imaginary part. In this case the structure theorem may be stated in a more informative form as follows:

Theorem. Let $A$ be a spectral operator on a Hilbert space $H$ whose imaginary part is completely continuous. Then $H$ is decomposed in an algebraic direct sum $H=H_{0}+H_{1}+H_{2}+\cdots$ of a countable family of invariant subspaces of $A$ such that $A \mid H_{0}$ is a scalar type spectral operator with real spectrum and each $A \mid H_{i}(i=1,2, \ldots)$ has the form $\lambda_{i} I_{i}+N_{i}$, where $I_{i}$ is the identity operator on $H_{i},\left\{N_{i}\right\}$ is a sequence of quasi-nilpotent completely continuous operators approaching zero in norm and $\operatorname{Im} \lambda_{i} \rightarrow 0$ as $i \rightarrow \infty$.

By an operator we always understand a bounded linear transformation on a Hilbert space. A von Neumann algebra means a selfadjoint operator algebra with the identity operator which is closed in the weak topology, and the set of operators each of which commutes with every operator in a von Neumann algebra $M$ is called the commutant of $M$ and denoted by $M^{\prime}$. It is well known that the commutant $M^{\prime}$ is again a von Neumann algebra and $M=M^{\prime \prime}$. A factor means a von Neumann algebra whose center consists of scalar multiples of the identity operator.

1. Let $A$ be an operator on a Hilbert space $H$ whose imaginary part $\operatorname{Im} A=\left(A-A^{*}\right) / 2 i$ is completely continuous and let $R(A)$ be the

Received by the editors September 5, 1968.

1 This research was partially supported by the National Science Foundation (NSF Contract No. GP-8291). 
von Neumann algebra generated by $A$. Then we have proved in $[\mathbf{5}$, Theorem 1] that $A$ is decomposed by a countable family of mutually orthogonal central projections $P_{0}, P_{i}(i \in I)$ in $R(A)$ into the form

$$
A=A_{0} \oplus \sum_{i \in I} \oplus A_{i},
$$

where $A_{0}=A \mid P_{0} H$ is selfadjoint and each $A_{i}=A \mid P_{i} H$ is primary in the sense that it generates a factor. It will be shown that the algebraic structure theorem cited above implies essentially the theorem. Now let us recall that an operator $S$ on Hilbert space is said to be of scalar type if $S$ is similar to a normal operator, and a spectral operator is the sum $S+N$ of a scalar type operator $S$ and a quasi-nilpotent operator $N$ commuting with $S$. For the details of the theory of spectral operators, we should refer to the paper of N. Dunford [3]. Furthermore, we should keep in mind that if a spectral operator has the completely continuous imaginary part, then its radical part is completely continuous as shown in [4, Theorem 1].

Let $A$ be a spectral operator on $H$ with completely continuous imaginary part. To apply the algebraic structure theorem to $A$ it seems to be quite relevant that we consider the case where its scalar part is normal. Let $T$ be an invertible operator on $H$ which transforms $A$ to a spectral operator $\tilde{A}$ whose scalar part $\tilde{S}\left(=T S T^{-1}\right)$ is normal. Then it seems that a question of whether or not $\operatorname{Im} \tilde{A}$ is completely continuous is nontrivial. However, by the aid of the spectral properties of operators with completely continuous imaginary part, it is possible to show that $\operatorname{Im} \tilde{A}$ is also completely continuous. For this purpose we may assume that $A$ is of scalar type because of the fact that the radical part is completely continuous. Let us recall first that every nonreal point in the spectrum $\sigma(A)$ is a proper value of $A$, the set of nonreal points $\{\lambda\}$ is at most countable and $\{\operatorname{Im} \lambda\}$ has no point of accumulation except possibly zero; and so $\sigma(\widetilde{A})$ has the same spectral properties. Let $\lambda$ be a nonreal point in $\sigma(A)$ and let $H_{\lambda}$ denote the generalized proper subspace of $A$ corresponding to $\lambda$, i.e., $H_{\lambda}=\left\{\varphi \in H \mid(A-\lambda I)^{n} \varphi=0\right.$ for some positive integer $\left.n\right\}$. Then, as seen in [2], $H_{\lambda}$ is finite dimensional. Thus $\widetilde{H}_{\lambda}=T H_{\lambda}$ is also finite dimensional, and moreover it must be a proper subspace of $\tilde{A}$ since $\tilde{A}$ is normal in this case. Denote by $\left\{\lambda_{n}\right\}$ the countable family of nonreal points in $\sigma(\tilde{A})(=\sigma(A))$ and set $K=\sum_{n} \oplus \tilde{H}_{\lambda_{n}}$. Since each $\tilde{H}_{\lambda_{n}}$ is finite dimensional and $\operatorname{Im} \lambda_{n} \rightarrow 0$ provided that $\left\{\lambda_{n}\right\}$ is infinite, $\tilde{A} \mid K$ has the completely continuous imaginary part. On the other hand, it is clear that $\tilde{A} \mid H \ominus K$ is selfadjoint. Consequently $\operatorname{Im} \tilde{A}$ is completely continuous. 
2. We are now in position to prove the theorem.

Proof of Theorem. First we observe that the scalar part $S$ of $A$ is in the von Neumann algebra $R(A)$ and the radical part $N$ of $A$ is also in $R(A)$. As is well known, if an operator $T$ commutes with $A$, then it commutes with $S$. This means that every operator in the commutant $R(A)^{\prime}$ of $R(A)$ commutes with $S$ and hence $S$ belongs to $R(A)^{\prime \prime}=R(A)$.

Following the foregoing treatment, we may assume without loss of generality that the scalar part $S$ is normal. By applying the algebraic structure theorem to the operator $A, A$ is decomposed by a countable family of central projections $P_{0}, P_{i}(i \in I)$ in $R(A)$ into the form

$$
A=A_{0} \oplus \sum_{i \in I} \oplus A_{i}
$$

where $A_{0}=A \mid P_{0} H$ is selfadjoint and each $A_{i}=A \mid P_{i} H$ is primary. Here, keeping in mind that $P_{i}$ is central, it is easily seen that $S_{i}$ $=S \mid P_{i} H$ is a normal operator belonging to $R\left(A_{i}\right)$ and $N_{i}=N \mid P_{i} H$ is a quasi-nilpotent completely continuous operator in $R\left(A_{i}\right)$. Since $S_{i}$ commutes with $A_{i}$, the normality of $S_{i}$ implies that $S_{i}$ commutes with $A_{i}{ }^{*}$, so that $S_{i}$ belongs to the center of $R\left(A_{i}\right)$. Thus $S_{i}$ must be a scalar multiple $\lambda_{i} I_{i}$ of the identity operator $I_{i}$ on $P_{i} H$ for each $i \in I$, and so each $A_{i}$ has the form $\lambda_{i} I_{i}+N_{i}$. Moreover, since $N$ $=N_{0} \oplus \sum_{i \in I} \oplus N_{i}\left(N_{0}=N \mid P_{0} H\right)$ and $N$ is completely continuous, we have $\left\|N_{i}\right\| \rightarrow 0$ in case $I$ is infinite. Therefore, setting $H_{0}=P_{0} H$ and $H_{i}=P_{i} H$, we complete the proof.

REMARK. In proving the structure theorem for a spectral operator $A$ satisfying the condition that $A A^{*}-A^{*} A$ is completely continuous, our treatment is still available. Indeed, the algebraic structure theorem for such an operator, which is a direct analog to our result employed here, may also be established (see H. Behncke [1]).

\section{REFERENCES}

1. H. Behncke, Structure of certain nonnormal operators, J. Math. Mech. 18 (1968), 103-107.

2. M. S. Brodskil and M. S. Livšic, Spectral analysis of non-self-adjoint operators and intermediate systems, Usephi Mat. Nauk. 13 (1958), 3-85; English transl., Amer. Math. Soc. Transl. (2) 13 (1960), 265-346.

3. N. Dunford, $A$ survey of the theory of spectral operators, Bull. Amer. Math. Soc. 64 (1958), 217-274.

4. J. Schwartz, On spectral operators in Hilbert space with compact imaginary part, Comm. Pure Appl. Math. 15 (1962), 95-97.

5. N. Suzuki, The algebraic structure of non self-adjoint operators, Acta Sci. Math. 27 (1966), 173-184.

University of California, Irvine 